\title{
Silicate, ruby, opal - Why gas giants keep their jewels in the atmosphere
}

\author{
Christiane Helling \\ ${ }^{1}$ SUPA, School of Physics \& Astronomy, University of St Andrews, North Haugh, St Andrews, \\ KY16 9SS, Scotland, UK \\ email: Christiane.Helling@st-andrews.ac.uk
}

\begin{abstract}
Giant gas-planets - and brown dwarfs - form dust clouds in their atmospheres which are made of a variety of gemstone-like and possible liquid materials. Our theoretical approach, where we calculate homogeneous nucleation, heterogeneous growth/evaporation, gravitational settling, and element consumption for composite dust grains, allows to access the evolution of the dust complex in the cloud, and hence also the elements remaining in the gas phase. The cloud formation process is imprinted into these remaining elements. Following a $(T, p)$ trajectory into the atmosphere we observe that 1 . metals disappear, 2. dust forms, 3. metals re-appear, 4 . dust disappears. For the first time, our kinetic cloud formation approach is coupled with an 1D atmosphere simulation and, hence, synthetic spectra can be produced based on detailed cloud micro-physics. Results are demonstrated for metal-poor gas giants and the strong influence of the dust modelling on alkali-line profile is shown.
\end{abstract}

Keywords. astrochemistry, radiative transfer, methods: numerical, stars: atmospheres, stars: low-mass, brown dwarfs, planets: atmospheres

\section{Introduction}

Cloud formation has a distinct influence on the atmospheres of substellar objects like giant gas planets and brown dwarfs, which in fact, share the basic mechanisms of cloud formation and micro-physics. Cloud formation is chemically (and physically) a kinetic process where a first surface has to form out of a gas phase (seed formation rate $J_{*}$ Fig. 1) †. This seed formation requires a considerable super-cooling, and a variety of other materials is already thermally stable at these temperatures. The seeds provide the surface where chemical surface reactions can grow a grain mantle made of these already thermally stable compounds, and the size of the grain increases rapidly due to gasgrain surface reactions. $\left(\langle a\rangle\right.$ in Fig. 1). The bigger the grains, the faster they fall $\left(\left\langle v_{\mathrm{dr}}\right\rangle\right.$ in Fig. 1) until the grain materials become thermally unstable at higher temperatures inside the atmosphere. The grain size decreases now due to evaporation and elements previously locked into the grains at higher altitudes are set free in deeper atmospheric layers. Convection is acting as transport mechanism on a certain time scale $\left(\tau_{\text {mix }}\right.$ in Fig. 1$)$ to bring up fresh material which keeps the cycle of dust formation running, producing the cloud in a substellar atmosphere.

$\dagger$ The seed formation process can be neglected in terrestrial planets since here wind sweeps up dust from the ground into the atmosphere. However, the actual rate, which determines the size and the number of the droplets, is still debated. 


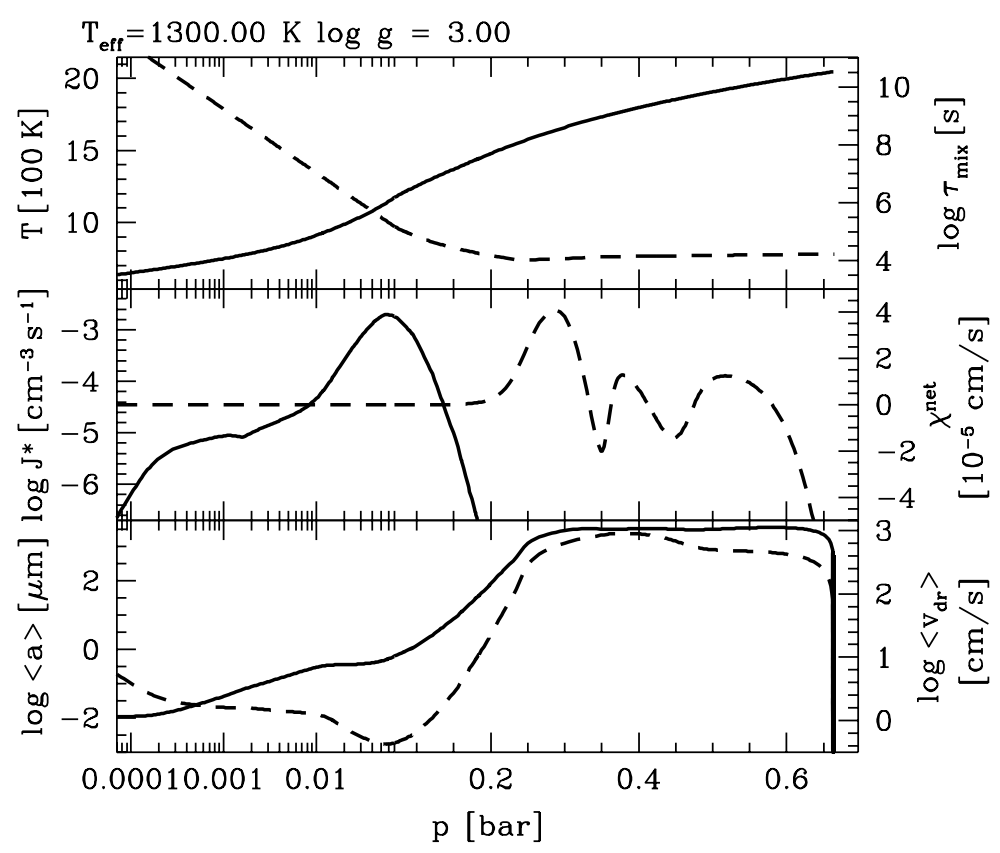

Figure 1. Dust cloud structure in a giant gas planet of $\mathrm{T}_{\text {eff }}=1300 \mathrm{~K}, \operatorname{logg}=3.0,[\mathrm{M} / \mathrm{H}]=0.0$. Top panel: Prescribed ( $\left.T, p, \tau_{\mathrm{mix}}\right)$-profile, Middle panel: nucleation rate $J_{*}$ (left), net growth velocity $\chi^{\text {net }}$ (right), Bottom panel: mean grain size $\langle a\rangle$ (left), drift velocity $\left\langle v_{\mathrm{dr}}\right\rangle$ (right).

\section{Dust model}

We model homogeneous seed formation (nucleation), heterogeneous growth, evaporation, and drift (gravitational settling) of dirty dust particles in a quasi-static atmosphere by using the moment method (Gail \& Sedlmayr 1988; Dominik et al 1993; Woitke \& Helling 2003, 2004; Helling \& Woitke 2006; Helling, Woitke \& Thi 2008). We consider the formation of compact spherical grains out of an oxygen-rich gas by the initial nucleation of $\mathrm{TiO}_{2}$ seed particles, followed by the growth of a dirty mantle. The moment and elemental conservation equations are evaluated for given $\left(T, \rho, v_{\text {conv }}\right)$ either for a prescribed static model atmosphere structure (Helling, Woitke \& Thi 2008) or inside a iterative solution of the radiative transfer problem (Helling et al. 2008, Dehn et al. 2007). Our dust model calculates the amount of condensates, the mean grain size $\langle a\rangle$, the parameterised grain size distribution function, and the volume fractions $V_{\mathrm{s}}$ of each materials as a function of height $z$ in the atmosphere. 12 solids $\left(\mathrm{MgSiO}_{3}[\mathrm{~s}], \mathrm{Mg}_{2} \mathrm{SiO}_{4}[\mathrm{~s}], \mathrm{MgO}[\mathrm{s}]\right.$, $\mathrm{SiO}_{2}[\mathrm{~s}], \mathrm{SiO}[\mathrm{s}], \mathrm{Fe}[\mathrm{s}], \mathrm{FeO}[\mathrm{s}], \mathrm{FeS}[\mathrm{s}], \mathrm{Fe}_{2} \mathrm{O}_{3}[\mathrm{~s}], \mathrm{Al}_{2} \mathrm{O}_{3}[\mathrm{~s}], \mathrm{CaTiO}_{3}[\mathrm{~s}], \mathrm{TiO}_{2}[\mathrm{~s}]$ ) made of 8 elements ( $\mathrm{Ti}, \mathrm{Si}, \mathrm{Fe}, \mathrm{Al}, \mathrm{Mg}, \mathrm{O}, \mathrm{S}, \mathrm{Ca}$ ) are considered to form the grain mantle by 60 chemical surface reactions. We solve 19 stiff differential equations already in the dust and element conservation complex. All details of the dust model can be found in the above references. Note that the equation used here represent the stationary dust formation case omitting every time-dependence and, hence, can be applied in the framework of classical stellar atmosphere simulations. The time-dependent version of our dust models has by now been tested in turbulence calculations of brown dwarf atmospheres (Helling \& Woitke 2004, Helling 2005) and in winds of asymptotic giant stars (Woitke 2006). 


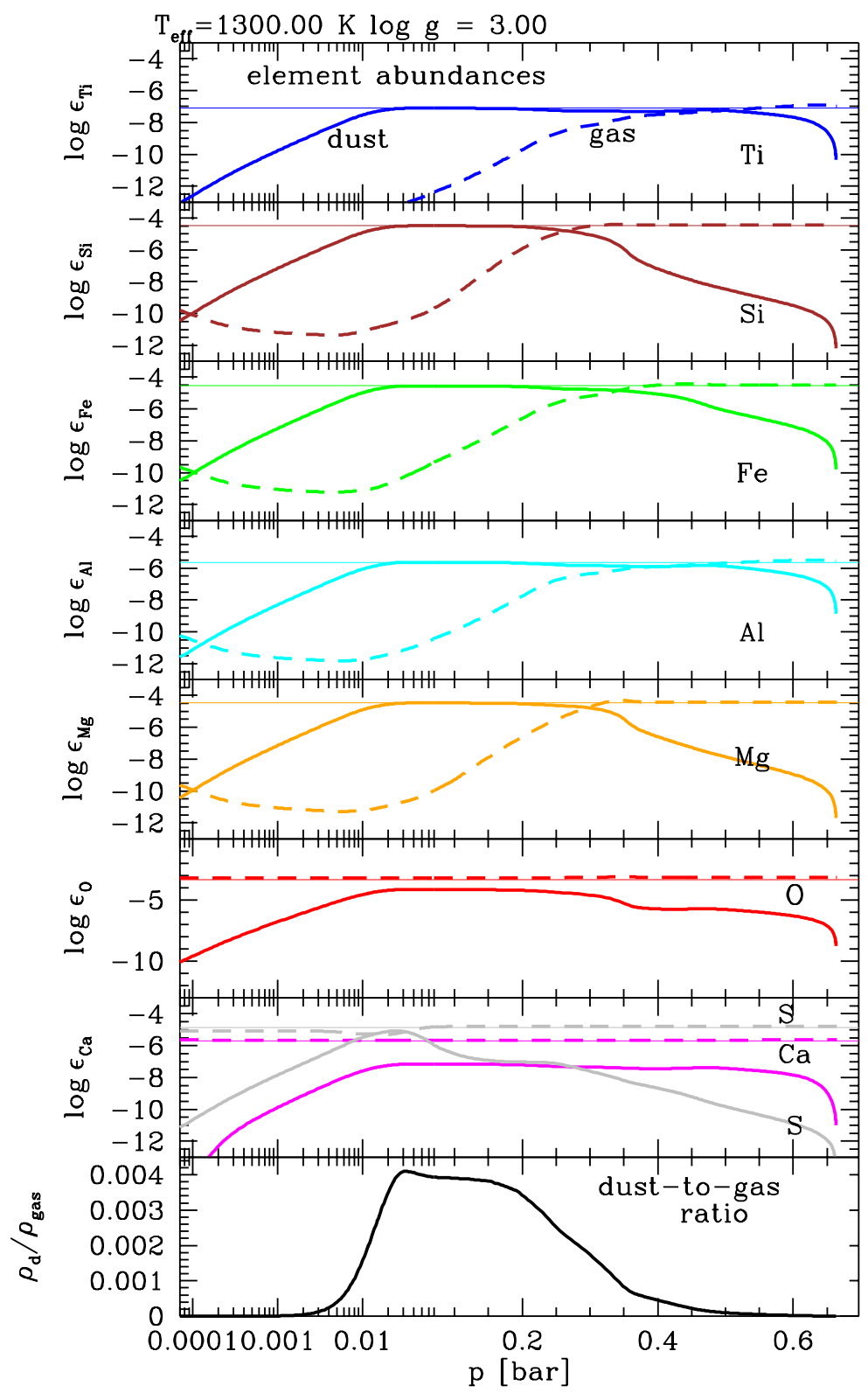

Figure 2. Abundances $\epsilon_{i}$ of the dust-forming elements (i=Ti, Si, Fe, Al, Mg, O, S, Ca) remaining in the gas phase (dashed), being bound in the dust (thick solid), and the solar values (thin solid) for $\mathrm{T}_{\mathrm{eff}}=1300 \mathrm{~K}, \operatorname{logg}=3.0,[\mathrm{M} / \mathrm{H}]=0.0$. Bottom panel: Dust-to-gas ratio $\rho_{\mathrm{d}} / \rho_{\text {gas }}$.

\section{Remaining gas-phase elements}

The amount of elements remaining in the gas phase is determined by the details of the dust formation like which compounds are condensing in which quantities, and the amount of condensed materials depends on the actual $(T, p)$-structure (Fig. 1). The material composition of the dirty dust grains forming the clouds and their grain size distribution throughout the cloud layer in a giant gas-planet has been presented in Helling (2007). A subtle feedback of the element abundances on the dust formation kinetics 


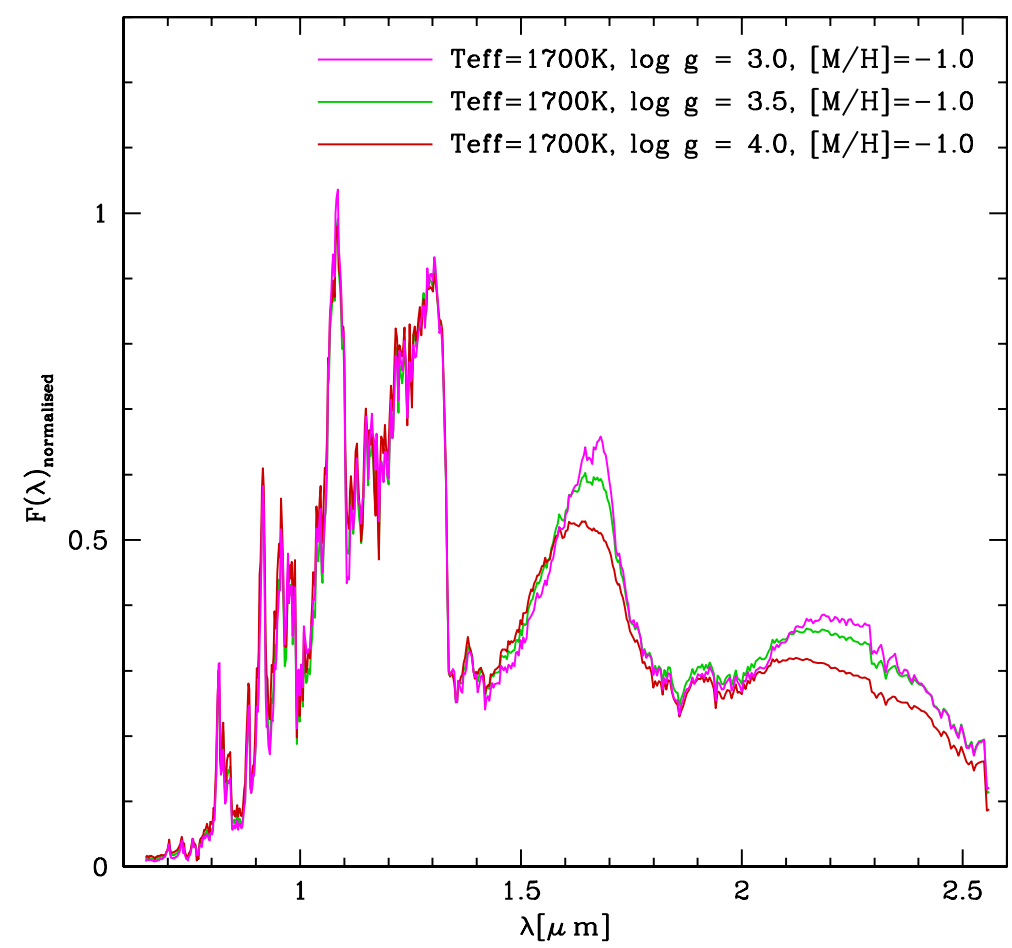

Figure 3. DRIFT-Phoenix synthetic spectra for metal-poor free-floating giant-gas planets for varying surface gravity: Only the flux in the $\mathrm{H} \& \mathrm{~K}$ bands decreases with increasing $\log g$.

exists and becomes most prominent when phase-equilibrium is asymptotically approached (see Helling, Woitke and Thi 2007, Sect. 4.2). Figure 2 demonstrates that the metallicity for each of the elements involved in the cloud formation changes with height in a unique way. It is impossible to assign a mean metallicity value to all the elements. At the top of the cloud layer the refractory elements disappear into the dust, second the dust appears, then the refractory elements re-appear, and then the dust disapears at the cloud base. The strongest gas-phase depletion appears above the cloud's dust maximum which can be seen by comparing the element abundances to the dust-to-gas ratio $\rho_{\mathrm{d}} / \rho_{\text {gas }}$ in Fig. 2 . Here, the abundances cannot be determined by phase-equilibrium arguments which is most obvious for $\mathrm{Ti}$ which is the limiting element involved in forming the seed particles.

\section{Coupling with $1 \mathrm{D}$ radiative transfer simulations}

Helling et al. 2008 (also Dehn et al. 2007) have combined our non-equilibrium dust formation model (DRIFT; Helling \& Woitke 2006, Helling, Woitke \& Thi 2007) with our general-purpose model atmosphere code (PhoEnix; Hauschildt \& Baron 1999) $\dagger$. In order to keep the computing time reasonable in the entire radiative transfer simulation, we consider only 7 solids $\left(\mathrm{MgSiO}_{3}[\mathrm{~s}], \mathrm{Mg}_{2} \mathrm{SiO}_{4}[\mathrm{~s}], \mathrm{MgO}[\mathrm{s}], \mathrm{SiO}_{2}[\mathrm{~s}], \mathrm{SiO}[\mathrm{s}], \mathrm{Al}_{2} \mathrm{O}_{3}[\mathrm{~s}], \mathrm{TiO}_{2}[\mathrm{~s}]\right)$ made of 5 different elements ( $\mathrm{Ti}, \mathrm{Si}, \mathrm{Al}, \mathrm{Mg}, \mathrm{O}$ ).

As one of our results, Fig. 3 shows synthetic spectra with sub-solar abundances and an effective temperature possible for gas-giant planets. We show how the spectra change in such low-metallicity objects with increasing surface gravity, log $g$, in the JHK spectral

$\dagger$ This is the same code as used by Allard et al. (2001) except for the dust chemistry (see Sect. 2) and line-profile calculations in Sect. 5. 


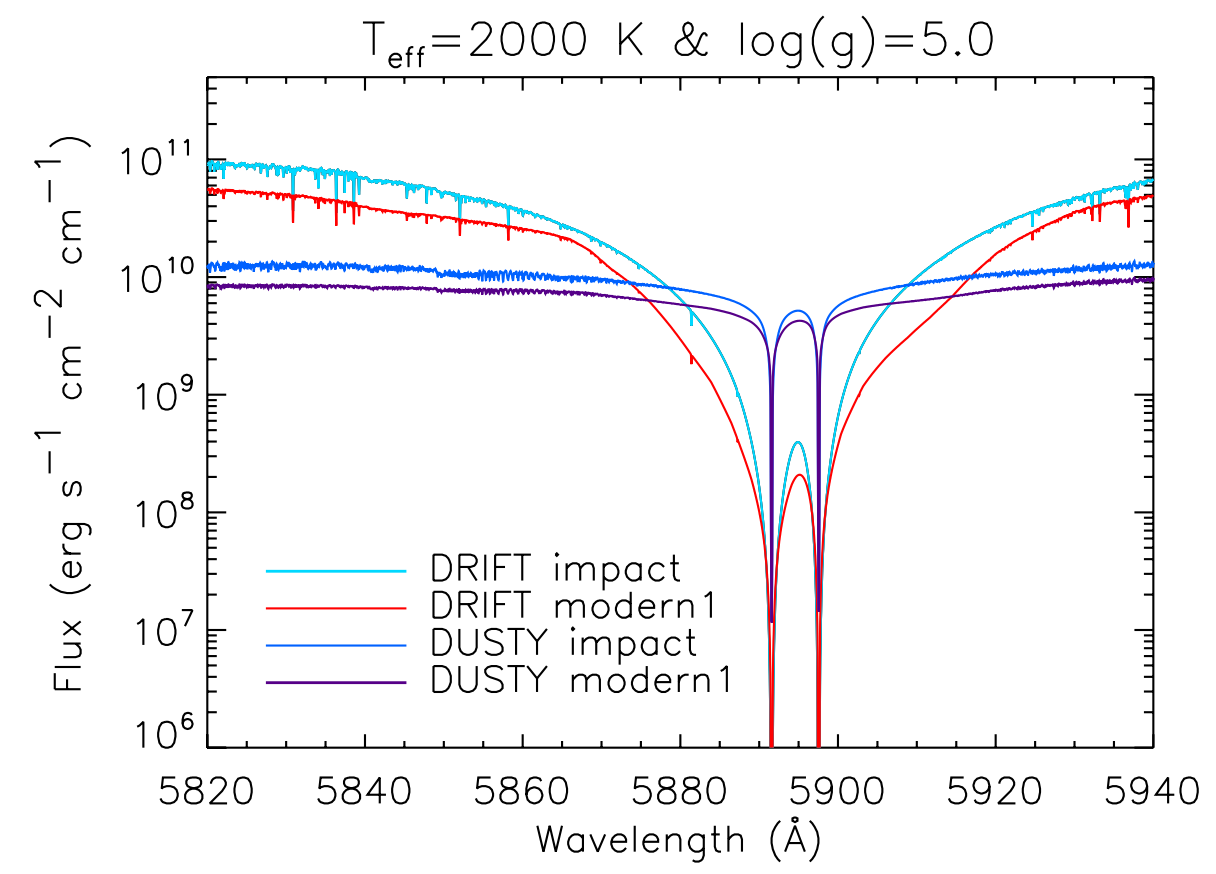

Figure 4. Synthetic spectra for the Na I doublet for DRIfT-Phoenix and Dusty-Phoenix simulations: DrifT-Phoenix produces much broader profiles and a higher pseudo-continuum. Note the different $\left(\mathrm{T}_{\text {eff }}, \log g\right)$ compared to Figs $1-3$.

region. The near-IR appears almost unaffected by the changing surface gravity, but the $\mathrm{H} \& \mathrm{~K}$ bands show a distinct $g$-dependences. The $\mathrm{H} \& \mathrm{~K}$ bands considerably flatten with increasing $\log g$ at such low metallicities in contrast to the more triangular shape of the lower-gravity cases. This flattening may help explaining the discrepancy between the GAIA models and the SINFONI observations of CT Chab (see Schmidt et al., this volume).

\section{Dust cloud modelling and $\mathrm{Na} I$ line profiles}

First studies are performed on the influence of dust modelling on the alkali line profile formation (Johnas 2007). Figure 4 shows results for the Na I doublet for two different dust models in the framework of PHOENIX atmosphere simulations: DRIFT has been shortly sketched in Sects. 1 \& 2, and DUSTy represents a limiting case where phaseequilibrium is assumed and the dust remains as opacity source in the atmosphere. Our results clearly demonstrates large differences in the width of the wings and hence, in the pseudo-continuum produced by the $\mathrm{Na} I$ lines. Also the depth of the absorption is different for model results of the two cloud approaches. Figure 4 furthermore demonstrates that the treatment of the dust clouds formation has a by far bigger influence on the synthetic spectrum than the treatment of the line profiles. However, the IMPACT-approach results in less absorption than the MODERN1-approach independent of the dust treatment (impact vs. modern1; for details see Johnas 2007, Johnas et al. 2007). 


\section{Conclusion}

Giant gas-planets keep their jewels in the atmosphere because they are continuously forming out of the gas phase under non-equilibrium conditions. The material composition of the individual grains gradually changes as the particles fall into deeper and hotter atmospheric layers (rain) which results in a cloud layer which mainly consist of silicates (opal? $\dagger$ ) at the top and high-temperature condensates like corundum (ruby? at the cloud base. This changing material composition is imprinted into the remaining gas-phase abundances and no mean element depletion due to cloud formation can be assumed in substellar objects. One indicator of the strong feedback onto the atmospheric thermodynamics are alkali line profiles. Their width and depth strongly depends on the the exact cloud formation treatment.

\section{Acknowledgement:}

ChH acknowledges an IAU travel grant.

\section{References}

Dehn M. 2007, PhD Thesis, University Hamburg

Dominik C., Sedlmayr E., Gail H.-P. 1993, A\& A 277, 578

Gail H.-P., Sedlmayr E. 1988, A\& A 206, 153

Hauschild P., Baron E. 1999, JCAM 109, 41

Helling Ch. 2005, eds. Kupka, Hillebrandt, 152; astro-ph/0506204

Helling Ch. 2007, ASPCS, eds. Fischer, Rasio, Thorsett, Wolszczan; arXiv:0711.3730v1

Helling Ch., Dehn M., Woitke P., Hauschildt P. 2008, ApJL submitted

Helling Ch., Woitke P. 2006, A\&A 455, 325

Helling Ch., Woitke P. 2004, A\&A 423, 657

Helling Ch., Woitke P. \& Thi W.-F. 2008, A\&A submitted

Johans C. M. S. 2007, PhD Thesis, University Hamburg

Johans C. M. S., Helling Ch., Woitke P., Hauschildt P. 2007, ASPCS, eds. Fischer, Rasio, Thorsett, Wolszczan

Ludwig H.-G., Allard F., Hauschildt P.H. 2006, A\& A 459, 599

Woitle P. 2006, A\&A 460, L9

Woitke, P. \& Helling, Ch. 2004, A\& A 414, 335

Woitke, P. \& Helling, Ch. 2003, A\& A 399, 297

$\dagger$ Hydrated silica $\left(\mathrm{SiO}_{2} \cdot n \mathrm{H}_{2} \mathrm{O}\right)$ is called opal.

$\ddagger$ Ruby and sapphire are $\mathrm{Al}_{2} \mathrm{O}_{3}$ crystals with metal inclusions: ruby $-\mathrm{Al}_{2} \mathrm{O}_{3}+\mathrm{Ti}$ (blue) and sapphire $-\mathrm{Al}_{2} \mathrm{O}_{3}+\mathrm{Cr}$ (red). 